La revue La revue pour l'histoire du CNRS

POURL'HISTOIRE DU CNRS

16 | 2007

L'expertise scientifique

\title{
Le changement climatique, une expertise entre science et politique
}

Amy Dahan

\section{OpenEdition}

Journals

Édition électronique

URL : https://journals.openedition.org/histoire-cnrs/1543

DOI : 10.4000/histoire-cnrs. 1543

ISSN : 1955-2408

Éditeur

CNRS Éditions

Édition imprimée

Date de publication : 3 avril 2007

ISBN : 978-2-271-06453-0

ISSN : $1298-9800$

\section{Référence électronique}

Amy Dahan, «Le changement climatique, une expertise entre science et politique », La revue pour I'histoire du CNRS [En ligne], 16 | 2007, mis en ligne le 26 mars 2009, consulté le 20 mai 2021. URL : http://journals.openedition.org/histoire-cnrs/1543; DOI : https://doi.org/10.4000/histoire-cnrs. 1543

Ce document a été généré automatiquement le 20 mai 2021.

Comité pour l'histoire du CNRS 


\title{
Le changement climatique, une expertise entre science et politique
}

\author{
Amy Dahan
}

1 En 1988, la question du réchauffement climatique global fait irruption sur la scène tant scientifique que géopolitique et médiatique. Après une sécheresse exceptionnelle aux États-Unis, quelques climatologues américains ou de l'Organisation météorologique mondiale alertent l'opinion sur le danger climatique. Une instance mondiale d'expertise, unique en son genre, le Groupe intergouvernemental d'étude du climat (GIEC), - en anglais International Panel on Climate Change (IPCC) -, est créée. Désormais, les recherches sur le changement climatique seront inséparables de l'ascension du problème sur la scène politique internationale, scandée en particulier par les conférences de Rio (1992), de Kyoto (1997), de Buenos Aires (2000), de New Delhi (2002), de Nairobi (2006), pour ne citer que les plus importantes.

Le cadrage du GIEC

2 Le cadrage général a été donné par les politiques. Mostafa Tolba (directeur de l'UNEP ${ }^{1}$ ) suggérait une organisation en trois groupes de travail (working groups, WG) parallèles qui s'occuperaient respectivement de science, des impacts du changement climatique (CC), des réponses à y apporter. Le choix des personnalités présidant les groupes et des responsables de divers sous-groupes fut pré-négocié entre les divers États; il reflétait tant les positions de pouvoir que la compétence technique. Il y a un apparent paradoxe à ce que, dans ce contexte hautement politique, le GIEC, organisation de plusieurs centaines de scientifiques, ait toujours attiré les meilleurs d'entre eux, et constamment produit des rapports qui engageaient leur crédibilité. Une réponse partielle réside dans l'existence d'une multiplicité d'acteurs politiques aux intérêts divergents (agences américaines concurrentes, UNEP, WMO ${ }^{2}$, pays différents); ce qui a conduit au scénario selon lequel ces acteurs abandonnent en quelque sorte le contrôle du processus d'évaluation en nommant un scientifique indépendant de grande notoriété (en l'occurrence Bert Bolin) à la présidence du GIEC. Ils préconisent aussi une «participation universelle» au processus - en 2005, 192 représentants d'États y ont participé -, enfin, le respect de règles procédurales strictes et transparentes. 
3 Les rapports seront préparés par des équipes de rédacteurs, durement sélectionnés sur la base de leur compétence scientifique (réputation, publications). Le processus de " referee » est très long et très lourd: les rédactions des chapitres devront passer par deux stades d'examen et de réécriture, d'abord par des pairs scientifiques, ensuite par les pairs et les gouvernements. Les rapports finaux devront être adoptés en session plénière et seront accompagnés de résumés techniques et de "résumés pour décideurs »; ceux-ci devront être approuvés ligne par ligne.

Les climatologues en pointe

4 Dans les premières années de fonctionnement du GIEC, alors que la réalité du changement climatique est encore l'objet de vives controverses, les scientifiques $\mathrm{du}$ premier groupe sont incontestablement très en pointe. S'appuyant sur une longue tradition de recherches, leurs modèles de circulation générale - seuls outils qui permettent de se projeter quantitativement dans le futur -, jouent un rôle crucial. Dès le premier rapport de 1990, à l'aide des modèles dans lesquels ils introduisent les concentrations de gaz à effet de serre, croissantes selon l'hypothèse du «business as usual », les climatologues prédisent un accroissement de la température moyenne du globe de $0,3{ }^{\circ} \mathrm{C}$ par décennie au cours du XXI siècle. Ils insistent toutefois sur les incertitudes (concernant les sources et les puits de gaz à effet de serre, le rôle des nuages, etc.).

5 En 1995, le deuxième rapport fait état d'avancées considérables dans la compréhension de divers aspects physiques du problème, et dans le développement de modèles couplant l'atmosphère avec les océans et les glaces. Le consensus scientifique que reflète le résumé du rapport identifie cette fois clairement une «influence humaine discernable au-delà de la variabilité naturelle ${ }^{3}$ (une petite phrase qui a exigé de très longues discussions) et un changement climatique déjà intervenu au cours du $\mathrm{XX}^{\mathrm{e}}$ siècle, tout en soulignant toujours un certain nombre d'incertitudes.

6 L'instauration du processus politique conduit insensiblement à une modification des rapports d'influence des trois groupes de travail. Le premier groupe continue à jouer un rôle déterminant, avec à sa tête des figures charismatiques comme Bert Bolin, Robert Watson, ou John Houghton. En 1990 comme en 1995, ou encore en 2001, les résumés des climatologues représentent les éléments majeurs des rapports d'évaluation, repris et commentés dans l'opinion publique car ils donnent les chiffres des prédictions physiques du changement climatique.

7 Kyoto (1997) marque les débuts de la montée en puissance du WG II, groupe de travail s'occupant des impacts, de la vulnérabilité et de l'adaptation au changement climatique. Il réunit des biologistes, des écologues, des hydrologues et a cherché à faire une place croissante aux scientifiques et ingénieurs des pays du Sud.

La co-construction entre science et politique

8 A priori, la mission donnée par les politiques aux scientifiques s'inscrit dans un paradigme jugé classique de l'expertise : «mettez-vous d'accord, vous scientifiques, et ensuite, nous politiques, nous agirons ». Ce cadrage de séparation radicale est accepté par les scientifiques car il semble préserver leur sphère d'autonomie. Mais de fait, le modèle linéaire ne fonctionne jamais comme tel. Les historiens et sociologues ont montré qu'il est totalement inadéquat pour rendre compte des allers-retours complexes entre connaissances fondamentales et applications, ou des processus de " co-construction» du scientifique et du politique, notamment celui qui s'est déroulé depuis quinze ans dans le domaine du changement climatique. 
9 En effet, le rôle du GIEC ne s'est pas réduit à la seule préparation des grands rapports d'évaluation. Dans la première moitié des années 1990, une reconfiguration du régime climatique s'est clairement jouée, à la suite de l'insatisfaction des pays en développement qui voyaient principalement la question de l'effet de serre comme un problème de surconsommation des pays du Nord.Or, le GIEC ne se prononce pas sur cette question qui est hors de ses compétences. Dès 1992, la direction du GIEC lance la préparation de six rapports dits spéciaux ou techniques - sur les méthodologies d'évaluations nationales des émissions des gaz à effet de serre, sur des questions énergétiques et industrielles, sur des questions agricoles et forestières, sur des scénarios d'émissions, sur les technologies de séquestration du carbone... La publication de ces rapports a correspondu à la volonté de répondre de façon plus réactive aux demandes des politiques et d'alimenter directement le processus de décision.

10 Bien que le credo officiel, constamment réaffirmé par les présidents successifs du GIEC, est que l'organisation a seulement les moyens et la mission d'évaluer les recherches déjà existantes, il a contribué à reconfigurer la recherche en mettant en avant des questions jusque là peu considérées : rôle des sols et des forêts, prédictions régionales, vulnérabilité à la montée des eaux, rôle du cycle du carbone.

Économistes et scénarios du GIEC

11 Les économistes jouent un rôle d'experts dans les trois groupes du GIEC. Dans le premier, ils assument la position, minoritaire mais cruciale, de fournir des scénarios d'évolution de nos besoins et de nos productions, en bref de donner à imaginer des scénarios de croissance. Dans le second, ils ont à évaluer les impacts sociaux du réchauffement et dans le troisième, ils doivent inventer les instruments économiques et industriels les plus adéquats à mettre en place, comme ce fut le cas dans le protocole de Kyoto (1997).

12 Selon la tradition du calcul économique, les coûts associés aux pollutions et aux dommages sont à mettre en balance avec les coûts des politiques de réduction de l'effet de serre. Le problème ici est de déterminer la répartition optimale dans le temps des actions à entreprendre. Les dommages étant de nature très variée (capital naturel, capital économique, adaptation, risques sanitaires, etc.) et objets de grandes incertitudes, leur évaluation marchande est très difficile et sujette à vives controverses. Celles-ci se déploient en particulier autour de la question : quand et où agir contre le changement climatique? (Voir la controverse "When and where flexibility» dans Nature ${ }^{4}$ ).

13 Le troisième rapport du GIEC (2001) présente de manière assez détaillée les résultats des projections climatiques jusqu'à la fin du $\mathrm{XXI}^{\mathrm{e}}$ siècle. Ces projections ont été réalisées une fois franchie une étape préalable : déterminer pour la même période des scénarios d'évolution des «agents de forçage " que sont les gaz à effet de serre et les aérosols, travail qui relève ici des socio-économistes. Ceux-ci élaborent donc une série d'images possibles de l'avenir qui ont une cohérence interne en termes de démographie, de modes de développement économique, de choix sociaux et technologiques ${ }^{5}$. Ces images sont ensuite traduites principalement avec une seule variable globale ${ }^{6}$, la concentration de gaz carbonique dans l'atmosphère - soit le nombre de particules de carbone par volume. Cette dernière variable permet de passer à la température moyenne du globe qui s'est avérée plus simple à manipuler que 
d'autres indicateurs climatiques (par exemple les précipitations), choix qui présente évidemment un caractère réducteur.

Le GIEC a défini quatre grandes classes de scénarios de croissance A1, B1, A2, B2 qui se distinguent à la fois par leur "soutenabilité » (le A est associé à une convergence de clauses matérielles et productivistes, le B est associé à des clauses de développement durable) et par leur « ouverture » (le 1 est associé à des conditions de marché global, le 2 est marqué par un développement régional de type protectionniste). Un scénario est une configuration d'hypothèses (sur la démographie, la consommation, les technologies, etc.) qui s'intègrent en un tout cohérent de manière tant synchronique que diachronique. L'origine de la notion de scénario se situe dans les travaux issus de la dynamique des systèmes de Forrester et du Club de Rome des années 1970 et dans une réflexion récurrente et controversée sur les limites de la croissance. Elle a été reprise dans les années 1980 et 1990 dans des modèles dits d'évaluation intégrée, qui couplaient des modèles physiques très simplifiés avec des modèles économiques pour constituer des instruments de simulation au service de décideurs et de négociateurs.

À de multiples égards, il est assez remarquable que l'instance scientifico-politique du GIEC ait renoué, avec les termes d'un choix fondamental pour notre avenir relatif à nos modes de développement (durable ?), de croissance (?), à nos conceptions des rapports d'échange entre les diverses parties du globe, tout particulièrement entre le Nord et le Sud. Ces scénarios entrent maintenant dans les simulations climatiques du XXI ${ }^{e}$ siècle, que divers groupes et sous communautés scientifiques mettent en œuvre pour le prochain rapport du GIEC, dont la publication est prévue en 2007. Comment ne pas regretter alors que les hypothèses implicites qui constituent la base des scénarios fassent si peu l'objet de débats publics ou politiques, y compris chez les chercheurs en sciences sociales?

Et demain?

16 La place originelle assignée au GIEC, la structuration qu'il a adoptée, l'expertise réflexive qu'il a réussie à promouvoir (partiellement due aux pressions politiques pour une déconstruction des assertions scientifiques), l'attention qu'il a accepté de porter aux préoccupations des pays du Sud, autant de facteurs qui ont fini par lui assurer le respect tant des scientifiques que des politiques. Les va-et-vient qui s'effectuent, sous ses auspices, entre les divers WG, entre les divers intérêts, plus généralement entre science et politique, en font le véritable fer de lance du régime climatique.

17 Contrairement à l'épistémologie traditionnelle du modèle d'expertise que pourtant il revendique, le GIEC a joué un rôle essentiel à l'articulation des deux ordres du scientifique et du politique qui ont avancé conjointement, en interaction l'un avec l'autre, y compris en l'absence de consensus général au sein de chacun d'eux ${ }^{7}$.

\section{NOTES}

1.United Nations Environment Programme.

2.World Meteorological Organization. 
3.Le résumé du rapport dit : "the balance of evidence suggests a discernible human influence on global climate".

4.Wigley, T.M.L., Richels, R. and Edmonds, J.A., 1996: Economic and environmental choices in the stabilization of atmospheric $\mathrm{CO}_{2}$ concentrations. Nature 379, 240-243. La controverse s'est poursuivie dans plusieurs livraisons de Nature en 1997 avec l'intervention de plusieurs auteurs dont William D. Nordhaus et Minh Ha-Duong. 5.Ces scénarios ont été élaborés sans postuler l'intervention explicite de politiques générales en matière de climat et ne prennent pas en compte les objectifs d'émissions fixés par le protocole de Kyoto, même s'ils postulent à des degrés divers l'effet de préoccupations gouvernementales, par exemple le développement de technologies peu consommatrices d'énergie.

6.Dans le troisième rapport de 2001, on a introduit quatre autres variables qui sont les concentrations d'autres gaz à effet de serre que le gaz carbonique. Et on parle d'» équivalent carbone ».

7.Il est en passe de devenir l'idéal type pour d'autres projets internationaux d'assessment : biodiversité (Millenium Ecosystem Assessment), eau (World Water vision), agriculture (International Assessment of Agricultural Science and Technology of Development).

\section{RÉSUMÉS}

Il existe dans le domaine du changement climatique, une instance intergouvernementale d'expertise, très singulière qui joue un rôle déterminant et tend à devenir un idéal type pour d'autres projets d'expertise internationaux. Quel en est le mode de fonctionnement?

Comment se positionne-t-elle entre science et politique? Amy Dahan propose des éléments de réponse.

A remarkable intergovernment authority exists in the area of the climate change. It plays a deciding role and tends to become a model for other international assessment projects. What is its operating mode? What is its place between science and politics? Amy Dahan suggests some clues.

INDEX

Mots-clés : expertise, politique, changement climatique, climat, GIEC, réchauffement

\section{AUTEUR}

\section{AMY DAHAN}

Amy Dahan est historienne des sciences, directrice de recherche au CNRS, au Centre Alexandre Koyré. Elle a conduit depuis 2003 un projet de recherche pluridisciplinaire sur les modèles du changement climatique. 